\section{$\underset{\substack{\text { hommes } \\ \text { \& migrations }}}{ }$}

\section{Hommes \& migrations}

Revue française de référence sur les dynamiques

migratoires

1308 | 2014

Les Paris des migrants

\title{
Les Paris des migrants
}

\section{Marie Lazaridis et Serge Weber}

URL : http://journals.openedition.org/hommesmigrations/2983

DOI : 10.4000/hommesmigrations.2983

ISSN : 2262-3353

\section{Éditeur}

Musée national de l'histoire de l'immigration

\section{Édition imprimée}

Date de publication : 1 octobre 2014

Pagination : 8-10

ISBN : 978-2-919040-29-2

ISSN : 1142-852X

\section{Référence électronique}

Marie Lazaridis et Serge Weber, «Les Paris des migrants », Hommes \& migrations [En ligne], 1308 | 2014, mis en ligne le 01 octobre 2017, consulté le 03 mai 2019. URL : http://

journals.openedition.org/hommesmigrations/2983; DOI : 10.4000/hommesmigrations.2983 


\section{LES PARIS DES MIGRANTS}

Par MARIE LAZARIDIS, enseignante, spécialisée dans la scolarisation des enfants de migrants dans l'académie de Paris, et SERGE WEBER, géographe, université Paris-Est Marne-la-Vallée, laboratoire Analyse comparée des pouvoirs.

Paris ville cosmopolite? C'est une évidence. Aucune grande métropole ne s'est constituée sans de multiples immigrations : partout, la croissance urbaine et la métropolisation sont le résultat d'une attractivité migratoire qui est le socle premier de l'attractivité économique. Ce numéro explore les différentes facettes et temporalités d'un Paris fait et vu par les migrants, qu'ils soient asiatiques, africains, américains ou russes, sans-papiers, commerçants, ouvriers, étudiants, musiciens ou écrivains, attachés ou non à des quartiers où les centralités commerciales sont progressivement mises en tourisme. Pour nombre de migrants, l'effervescence humaine et la monumentalité de l'espace parisien ont construit une image quasiment iconique de la "Ville-lumière", associant romantisme, bohème, luxe, plaisirs, réussite et rêves. En retour, cette vie intense des espaces publics parisiens tout autant que les grands travaux d'embellissement ont été le produit des nouveaux arrivants, comme l'ont rappelé les principaux ouvrages retraçant l'histoire, la sociologie et la géographie de la capitale ${ }^{1}$. Les immigrants n'ont cessé d'animer marchés, rues commerçantes, entreprises, ateliers, usines, spectacles, vie intellectuelle et culturelle : pas de cafés ni de brasseries, ces hauts lieux de la sociabilité parisienne, sans les Auvergnats, les Aveyronnais, les Kabyles ni, plus récemment, les Wenzhous ${ }^{2}$; pas d'avant-garde sans les Russes, les Allemands, les Polonais, les Américains du Nord comme du Sud, pas de haute couture ni de prêt-à-porter sans les émigrants d'Afrique du Nord, d'Europe centrale et balkanique, du Levant, d'Anatolie ni du reste de l'Asie ${ }^{3}$; pas de première région industrielle de France sans les Algériens, les Marocains, les Portugais, les Italiens, les Africains de pays situés au sud du Sahara, les Yougoslaves ni tant d'autres encore. Et ce sont ces nouveaux venus qui ont littéralement construit la ville: pas de percées haussmanniennes, ni de métro, ni de périphérique, ni aucun des nouveaux aménagements qui continuent de renforcer le rayonnement de l'agglomération, sans ces ouvriers recrutés

1. Jacqueline Beaujeu-Garnier, Jean Bastié (dir.), Atlas de Paris et de la région parisienne, Paris, Berger-Levraut, 1967 ; Jean-Luc Pinol (dir.), Atlas des Parisiens, de la Révolution à nos jours, Paris, Parigramme, 2009 ; Éric Hazan, L'Invention de Paris. Il n'y a pas de pas perdus, Paris, Seuil, 2012 ; David Harvey, Paris, capitale de la modernité, Paris, Les Prairies ordinaires, 2012 ; Anne Monjaret (dir.), Le Paris des ethnologues, des lieux, des hommes. Ethnologie française, vol. $42 \mathrm{n}^{\circ}$ 3, 2012. 2. Anne Steiner, Sylvaine Conord, “Portrait d'un bistrot des faubourgs : Le Mistral”, in Ethnologie française, vol. 42, n 3, 2012, pp. 471-481. 3. Mirjana Morokvasic, "Immigrants in the Parisian garment industry", in Work, Employment \& Society, 1987, vol. 1, pp. 441-462 ; Solange Montagné-Villette, Le Sentier, un espace ambigu, Paris, Elsevier Masson, 1990 ; Nancy Green, Du Sentier à la Septième Avenue, la confection et les immigrés, Paris, New York, 1880-1980, Paris, Seuil, 1998. 
en masse, d'abord dans le reste de la France, notamment en Limousin ${ }^{4}$, ensuite dans les colonies puis dans les anciennes colonies, les pays voisins ou lointains. En ce sens, l'action des migrants qui ont fait Paris a longtemps été occultée, faisant écho à l'histoire des ouvriers parisiens depuis le début du XIXe siècle ${ }^{5}$. La diversité sociale de l'immigration a été longtemps ignorée : élites internationales entrepreneuriales ou consommatrices, enfants des classes moyennes animant les creusets intellectuels et universitaires sur les bancs des facs et des bibliothèques, minorités cherchant refuge au gré de la dislocation des empires et de la construction d'États-nations plus ou moins tolérants, opposants politiques, ce sont autant de mondes qui se sont reconstitués, transformés, mélangés dans le formidable creuset parisien et francilien, fortement influencés par des logiques migratoires internes et spécifiques ${ }^{6}$. Chacun de ces mondes a laissé ses traces encore vivantes en des lieux emblématiques dont aucun n'est figé ni exclusif et qui participent à la Ville-monde ${ }^{7}$ d'aujourd'hui, celle qui sétend au-delà des barrières du périphérique. Le Sentier, Belleville, le Triangle de Choisy, Barbès et Château-Rouge, Château-d'Eau, le faubourg SaintDenis, le faubourg du Temple, La Chapelle, Aubervilliers, Saint-Denis, La Courneuve, Bobigny, Torcy et d'autres centralités sont au Paris d'aujourd'hui, à leur manière, ce que Montparnasse, le Quartier latin, le Marais ou l'îlot Chalon ont été par le passé8. C'est là qu'il convient de distinguer entre espace "habité" et espace résidentiel : ce qui est visible dans le paysage urbain ne l'est pas toujours dans le recensement de la population et inversement, ce qui est visible dans les statistiques des résidents ne se traduit pas toujours par un marquage des lieux. Aujourd'hui, l'attractivité a un prix, à commencer par celui du sol, ô combien précieux intra-muros, mais aussi de plus en plus au-delà du périphérique, où se combinent une amorce de gentrification dans les quartiers populaires et les projets de centralités prévus dans la future configuration du Grand Paris. L'embourgeoisement des beaux quartiers, la gentrification des quartiers anciennement populaires ${ }^{9}$, les changements stratégiques de la politique de la Ville dans la production et la transformation des logements sociaux depuis la loi relative à la solidarité et au renouvellement urbains de 2000, dite "loi SRU", et les suivantes ont alimenté dans l'espace résidentiel les mécanismes de sélection sociale et de relégation par la distance. Elles ont deux conséquences majeures. La première, c'est qu'on ne peut plus envisager "le Paris des migrants" sans le reste de l'agglomération : l'interdépendance entre les lieux de résidence et de fréquentation montre une géographie de l'immigration qui a changé d'échelle depuis un demi-siècle. La seconde, c'est que les processus de différenciation sociale, fortement contraints par le foncier, se font à l'échelle des îlots, voire des immeubles. La "société partagée" de mondes sociaux distants se joue pour partie dans les espaces publics et collectifs $^{10}$. L'immigration est donc un élément central du système productif de la ville, tant pour l'activité

\footnotetext{
4. Alain Corbin, Archaïsme et modernité en Limousin au XIXe siècle (1845-1880), Limoges, Presses universitaires de Limoges, 1999 (1 1 édition 1975). 5. Maurizio Gribaudi, Paris ville ouvrière, une histoire occultée (1789-1848), Paris, La Découverte, 2014 ; Gérard Noiriel, Les Ouvriers dans la société française, XIXe-XXe siècles, Paris, Seuil, 1986. 6. Gérard Salem, "De Dakar à Paris, des diasporas d'artisans

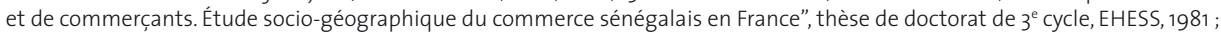
Jacques Barou, "Les immigrations africaines en France : des navigateurs au regroupement familial", in Revue française des affaires sociales, $n^{\circ}$ 1, 1993, pp. 191-205; Chloé Cattelain, Marylène Lieber, Claire Saillard, Sébastien Nguyen, "Les déclassés du Nord. Une nouvelle migration chinoise en France”, in Revue européenne des migrations internationales, vol. 21, n 3, 2005, pp. 27-52 ; MarieChristine Volovitch-Tavares, Portugais à Champigny. Le temps des baraques, Paris, Autrement, 2001 ; Pierre Kamdem, Camerounais en île-de-France. Dynamiques migratoires et stratégies d'intégration socio-spatiale différenciées, Paris, L'Harmattan, 2007; Catherine Servan-Schreiber, Vadooseven Vuddamalay (dir.), Hommes \& Migrations, n 1268-1269, "Diasporas indiennes dans la ville”, 2007 ; Leyla Sall, "Les champs commerciaux sénégalais à Paris : coprésences, luttes pour l'espace et stratégies commerciales au sein d'espaces urbains interstitiels", in Diversité urbaine, vol. 10, n¹, 2010, pp. 61-83. 7. Estelle Auguin, "Le Nouvel An chinois à Paris : théâtre d'économies ethniques", in Revue européenne des migrations internationales, vol. 20, n 3, 2004, pp. 75-87; Anne Hertzog, "La pagode vietnamienne de Linh Son à Joinville-le-Pont : centre d'une organisation territoriale discontinue", in Géographies et cultures, $\mathrm{n}^{\circ} 52$, 2004, pp. 38-54. 8. Véronique De Rudder, Michelle Guillon, Autochtones et immigrés en quartier populaire : du marché d'Aligre à l'îlot Châlon, Paris, L'Harmattan, 1987 ; Bernard Dinh, "Le faubourg Saint-Denis : un terrain marchand à l'épreuve de la diversité culturelle", Hommes \& Migrations, n 1280, 2009, pp. 106-117; Gaëlle Dequirez, "Processus d'appropriation et luttes de représentation dans le 'Little Jaffna' parisien”, in Revue européenne des migrations internationales vol. 26, n 2, 2010, pp. 95-116.
} 
de transformation que pour l'économie résidentielle, en particulier la production urbaine, la reproduction sociale ${ }^{11}$ et le commerce ${ }^{12}$ : les migrants font vivre Paris et les Parisiens. Les opportunités professionnelles, plus ou moins précaires, plus ou moins cantonnées à des niches économiques, leur permettent une insertion et des parcours de réussite - parfois au prix d'une précarité accentuée et prolongée qui confine pendant un temps à l'exploitation $^{13}$. Sans oublier que la précarité administrative fragilise bien des étrangers et les rend plus vulnérables sur le marché du travail, quand bien même les infrastructures d'accueil et d'accompagnement ne manquent pas à Paris ${ }^{14}$. À l'autre bout du système productif, c'est le capital qui est en jeu, et les migrants y participent aussi, de même qu'au marché du travail très qualifié ${ }^{15}$. Ce n'est qu'à la lumière de ces multiples prismes de différenciation des positions sociales qu'habiter la métropole parisienne est toujours pour les migrants ${ }^{16}$ un pari, autant de paris que de trajectoires et d'histoires individuelles.

9. Thérèse Saint-Julien, Renaud Le Goix (dir.), La Métropole parisienne. Centralités, inégalités, proximités, Paris, Belin, 2007 ; Michel Pinçon, Monique Pinçon-Charlot, Sociologie de Paris, Paris, La Découverte, 2008 ; Anne Clerval, Paris sans le peuple. La gentrification de la capitale, Paris, La Découverte, 2013. 10. Patrick Simon, "La société partagée. Relations interethniques et interclasses dans un quartier en rénovation, Belleville, Paris XXe", in Cahiers internationaux de sociologie, vol. XCVIII, 1995, pp. 161-190. 11. Laura Oso Casas, "La réussite paradoxale des bonnes espagnoles de Paris. Stratégies de mobilité sociale et trajectoires biographiques", in Revue européenne des migrations internationales, vol. 21, $\mathrm{n}^{\circ}$ 1, 2005, pp. 107-129; Caroline lbos, Qui gardera nos enfants? Les nounous et les mères, Paris, Flammarion, 2012. 12. Claire Zalc, Melting shops, une histoire des commerçants étrangers en France, Paris, Perrin, 2010. 13. Nicolas Jounin, Chantier interdit au public. Enquête parmi les travailleurs du bâtiment, Paris, La Découverte, 2008 ; Yun Gao et al. "De la migration au travail. L'exploitation extrême des Chinois(e)s à Paris", in Travail, genre et sociétés n 16, 2006, pp. 53-74. 14. Régis Bigot, "Le parcours d'une centaine de mineurs isolés roumains suivis par l'association Hors la rue et pris en charge par l'aide sociale à l'enfance de Paris", in e-migrinter, vol. 2, 2008, pp. 121-131. 15. Thierry Berthet, “L'immigrant aisé dans la ville", in Nicole Haumont, Jean-Pierre Lévy (dir.) La Ville éclatée. Quartiers et peuplement, Paris, L'Harmattan, 1998, pp. 145-159 ; Emmanuelle Santelli, “Cadres et entrepreneurs d'origine algérienne. Histoires familiales et itinéraires”, in Ville-École-Intégration Enjeux, n 113, 1998, pp. 118-135. 16. Elif Aksaz, “Immigration familiale turque et activités quotidiennes des femmes. Le souci de réputation dans une cité HLM de la banlieue parisienne", in Revue européenne des migrations internationales, vol. 22, n 3, 2006 pp. 155-177; Marie-Claude Blanc-Chaléard, "Immigration, vie politique et politique en banlieue parisienne, XIXe et XX' siècles", in Vingtième Siècle, n 46, 1995, pp. 184-188. 